\title{
Anderson transition and thermal effects on electron states in amorphous silicon
}

\author{
D. A. Drabold \\ Department of Physics and Astronomy, Condensed Matter \\ and Surface Science Program, Ohio University, Athens, Ohio 45701-2979, USA \\ http://www.phy.ohiou.edu/ drabold/drabold.htm
}

\begin{abstract}
I discuss the properties of electron states in amorphous Si based on large scale calculations with realistic several thousand atom models. A relatively simple model for the localized to extended (Anderson) transition is reviewed. Then, the effect of thermal disorder on localized electron states is considered. It is found that under readily accessible conditions, localized (midgap or band tail) states and their conjugate energies may fluctuate dramatically. The possible importance of non-adiabatic atomic dynamics to doped or photo-excited systems is briefly discussed.
\end{abstract}

\section{Introduction}

There have been a very large number of studies on electron states in amorphous materials, and especially amorphous silicon [1]. These computations have ranged from simplified tight-binding approaches to first principles methods using density functional theory in the local spin density approximation (LSDA) [2]. A difficulty with many calculations of electron states in amorphous $\mathrm{Si}$ is the usual challenge of obtaining a realistic starting model. Amorphous Si is well known to be almost entirely four-coordinated with fewer than one atom in $10^{3}$ being non-ideally coordinated. However, few if any models fabricated entirely with molecular dynamics (MD) (including first principles MD) have a defect concentration at all consistent with experiment. In particular, models obtained by a quench from the melt are particularly unrealistic, often with $10 \%$ or more coordination defects in a cell of order 100 atoms. Such a model does not posess a true optical gap, but rather just a deep minimum in the density of states near the Fermi level. This result is inconsistent with electron spin resonance (ESR) measurements and most other estimates of defect concentration in the material. Such models are probably best interpreted as poor metals rather than semiconductors, since there is so much banding between impurities that the defect states are fully extended through the supercell. The origin of this trouble is easy to ascertain: the liquid (a predominantly sixfold metal) is topologically quite distinct from amorphous (or cystalline) Si, and any rapid quench is certain to freeze in too many remnants of the liquid (such as overcoordination defects, which are more common than dangling bonds in most published MD simulations [2]). Because of the qualitative difference in liquid and amorphous topology, it is perhaps not surprising that a-Si cannot be formed experimentally from a quench from the liquid. We have shown elsewhere [2] that a proper description of defect wave functions (with localization con- sistent with estimates from ESR) requires both a realistic model (with a very small defect concentration less that $1 \%$ ) and LSDA, to estimate the spin localization of an isolated defect. Fortunately realistic models (meaning in uniform agreement with structural, vibrational and electronic/optical experiments) do exist, but are made using Wooten-Weaire-Winer [3] methods. An excellent set of these models (with 216, 512 and 4096 atoms) was produced by Djordjevic, Thorpe and Wooten [4]; we use these in the studies presented here.

It is now established that the midgap defect state is due to dangling bonds. The floating bond conjecture (that five-fold coordinated atoms play an important role in a-Si) has not been disproven, though any such states are shifted well toward the conduction band edge [2] and are not nearly so localized as the $\mathrm{sp}^{3}$ dangling bonds. If such floating bond defects exist, they might form resonances with the conduction tail states, which could have interesting implications for transport in n-type materials.

Less progress has been made on band tail states, because a good description of these requires models with large numbers (several hundred to thousands) of atoms. To obtain a qualitative understanding of the Anderson transition in a three-dimensional network we used a 4096 atom supercell model [4] (a cube of amorphous Si about $4 \mathrm{~nm}$ on a side). The band tail states have structure on a length scale several times the nearest neighbor distance (depending on the energy), and an enormous model (by the standards of electronic structure calculations) is essential to disentangle the "simple" features of the band tail tates in amorphous Si. In section 3 we describe the properties of these states.

Finally we discuss the electronic effects due to thermal disorder superposd on a topologically disordered network [5]. We observe from a straightforward thermal simulation of an amorphous Si network that well-localized states can have their energies modulated by tenths of an $\mathrm{eV}$ (interestingly, an order of magnitude larger than the thermal energy itself). Also, the states themselves can fluctuate spatially as we describe in section 4 below. This feature is of importance to transport, optical and doping studies.

\section{Methodology}

For the calculations of electronic tail states in the 4096 atom model, we used a tight-binding Hamiltonian and a sophisticated Lanzcos scheme to compute about 500 states near the optical gap [6] (the dimension of the Hamiltonian matrix is 16384). A technical point of some 
importance is that a "simple" Lanczos scheme designed for computing states near a selected energy, such as the folded spectrum method (computing states from a shifted and squared $\mathbf{H}$ matrix) is not adequate to compute more than about 20 states here because the spectrum becomes exceedingly dense for energies out of the middle of the gap. What is effective is a "shift and invert" strategy, which spreads the spectrum out effectively, enabling the reliable calculation of many more states. Thus, one computes the matrix $\mathbf{G}(E)=(E \mathbf{1}-\mathbf{H})^{-1}$; and performs an elementary Lanczos calculation on the matrix $\mathbf{G}$ (to obtain spectral information near $\mathrm{E} ; \mathbf{H}$ is the Hamiltonian matrix, $\mathbf{1}$ is the unit matrix). It is amusing that this method was apparently pioneered by engineers [7] instead of physicists, since the object $\mathbf{G}$ is so familiar to workers in condensed matter physics (it is the electronic Greens matrix!). It is not a priori obvious that the expense of inverting $(E \mathbf{1}-\mathbf{H})$ is worthwhile, but because $\mathbf{H}$ is sparse this is not prohibitively expensive, and the transformed spectrum is well conditioned for Lanczos (the eigenvalues of $\mathbf{G}(E)$ are well separated near $E$ even if the spectrum of $\mathbf{H}$ is dense near $E$ ).

For dynamical simulation, we use the methods of Sankey and coworkers [8] "Fireball96", which is discussed in the context of amorphous Si elsewhere [5].

\section{Model of the Anderson transition in amor- phous Si}

I have discussed the details of the computation of gap and band tail states in the 4096 atom model of amorphous Si elsewhere [6]; here I dwell upon the "physics" of the calculation. In a nutshell, we found that (1) states near the middle of the gap were exponentially localized (as one would expect); (2) for energies shifted from midgap toward the valence tail, there was a tendency to find system eigenstates with rather similar charge densities, and consisting approximately of two separated "blobs" of charge; (3) deeper into the valence tail we observed that the states still were discernibly "built" from clumps or clusters of charge, with a clear separation between these clumps; (4) deep into the valence tail we saw states that were well extended, essentially uniformly, through the cell. See Figs. 1 and 2 in Ref. [6]; (5) The states are very nonlocal away from midgap, in contradiction with the defect pool model; it is clear that this must happen since the states in the bands are extended; this computation shows that states are really well localized only within of order $\sim 0.6-0.7 \mathrm{eV}$ from midgap.

The preceding discussion suggests that an alternate basis or representation may be worthwhile to describe the electron states of a-Si, especially near the gap. As usual in quantum mechanics, one is at liberty to select any complete (or at least approximately complete) set to represent the quantum mechanical states. The choice is normally made on grounds of efficiency: basis functions are selected which capture much of the character of the eigenfunctions, so that only a small number of basis functions is required to faithfully represent the eigenfunctions. The classic example is the method of orthogonalized plane waves in band theory [9]. Likewise here, since the eigenvectors in the gap and near the tails are clearly composed of "lumps" or clusters of charge, a basis consisting of such cluster states is appealing.

I take the view that the cluster states are localized eigenfunctions which arise from idealized isolated defects. That is, one can imagine all possible distortions of a continuous random network. Select one such distortion (say a strained bond angle removed from the distribution of bond angles in the system). Suppose further that the strain is large enough to make the state bound (eg. localized) with exponential tail. Then the state has both a well defined energy and well defined extent in real space. Such a situation certainly exists in a-Si provided that the defect is really isolated (in space) from resonant defects. From this point of view, the highly localized states near the middle of the gap arising from single defects in large cells are examples of especially spatially compact cluster states. As one considers energies closer to the valence and conduction bands (in the band tail regions), it becomes difficult to compute the cluster states exactly, since the density of states increases (which implies that the number of cluster states is also greater) so that the probablity is large that the tails of resonant defects overlap. If there is such an overlap between resonant defects (clusters), simple quantum mechanics guarantees that eigenstates will consist of mixtures of cluster states with formation of "bonding and antibonding" levels. Another way of saying it is that we are in the curious position of: "Given the eigenvectors, what is the most localized basis with smallest number of elements which can be superposed to reproduce the eigenstates?" Such cluster states have much in common with our recent work on generalized Wannier functions in amorphous materials [10].

Our qualitative picture of the local to extended transition in a-Si, based on these calculations is the following: As severe distortions are rare, clusters stemming from such distortions are probably isolated from each other and if isolated, are localized energy eigenstates. For less severe distortion, the probability of occurrence increases, and the size of associated clusters is also larger. Then the chance of finding another cluster of similar energy in the neighborhood increases. As the distortion becomes less severe, eigenstates will consist of mixtures of two, three, or more clusters. At some point, clusters can always find "overlapping energy partners" and they mix together to enable electronic connectivity (extended eigenfunctions). This state of affairs can be identified with the "mobility edge".

With the preceding introduction, it is natural to write down a simple "theory" utilizing these ideas. A reasonable form for this model is:

$$
\hat{H}=\sum_{\alpha} E_{\alpha}|\alpha\rangle\left\langle\alpha\left|+\sum_{\alpha \beta(\alpha \neq \beta)}\right| \alpha\right\rangle \xi_{\alpha \beta}\langle\beta|
$$

where, basis state $|\alpha\rangle$ is a localized cluster (which may 
involve many atoms). In this representation, the basis functions are localized energy eigenstates with eigenvalue $E_{\alpha}$ of the Hamiltonian in the absence of other defects with which mixing occurs. $E_{\alpha}$ is determined by the distortion. In real amorphous solids, the cluster states may significantly overlap. $\xi_{\alpha \beta}$ represents the coupling between cluster $|\alpha\rangle$ and $|\beta\rangle$. In the spirit of Hückel theory, we can take: $\xi_{\alpha \beta} \sim\left(E_{\alpha}+E_{\beta}\right) S_{\alpha \beta} / 2$ for $S_{\alpha \beta}=\langle\alpha \mid \beta\rangle$. Then, in first order perturbation theory, the formation of eigenstates of $\mathbf{H}$ from these clusters becomes obvious; the first order correction to the zeroth-order (cluster) state $|\alpha\rangle$ is $\sum_{\beta \neq \alpha} \Gamma_{\alpha \beta}|\beta\rangle$, where $\Gamma_{\alpha \beta}=\left(E_{\alpha}+E_{\beta}\right) S_{\beta \alpha} / 2\left(E_{\alpha}-E_{\beta}\right)$. The strong mixing for small energy denominators $\left(E_{\alpha} \approx E_{\beta}\right)$, and the role of the overlap is indicated. For reasons that are apparent, we have called this the "resonant cluster proliferation" model of the Anderson transition [6].

Since the cluster basis states $|\alpha\rangle$ are not precisely known, no calculations have yet been attempted in this representation. It is however easy to imagine a calculation in which model cluster states are introduced with characteristic decay lengths; and the role of the amorphous net is largely encoded into $\xi_{\alpha \beta}$. Even in the absence of new explicit calculations, I assume that the "resonant cluster" model is useful to understanding the Anderson transition, and the role of thermal disorder in a topologically disordered network, as I describe next.

\section{Thermal modulation of electron states and en- ergies}

There is abundant experimental evidence that lattice vibrations play an important role in the dynamics of electrons in amorphous materials. Among other examples, Cohen and coworkers [11] observed a pronounced temperature dependence of the Urbach tails in a-Si:H (the conduction tails showing a very strong linear variation in decay parameter with temperature). Of course electrical conductivity is well known to be very temperature dependent, and usually has multiple distinct regimes according to different conduction mechanisms [12]. From this point of view it is unsurprising that the electron energies and states can be very time and temperature dependent. This point has been independently recognized by Arkhipov and Adriaennsens [13] in their studies of carrier transport.

We study thermal modulation of the eigenvalues and eigenvectors by tracking their (adiabatic) time development over the course of a few picosecond $\left(10^{-12} \mathrm{~s}\right)$ simulation. As illustrated in Fig. 1 in Ref. [5], there is strong time dependence of the LDA eigenvalues in the vicinity of the optical gap. The Fermi level is near the middle of the gap and several states near the Fermi level are appropriately described as band tail states. These are much like the states which would be responsible for conduction in doped a-Si:H. As in earlier work [14] there is a roughly linear relation between root mean square (rms) temporal fluctuation and temperature. As expected, the higher temperature simulation leads to larger excursions in the positions of the energy eigenvalues. Note for $300 \mathrm{~K}$ that the Lowest Unoccupied Molecular Orbital (LUMO) fluctuates in time by about $\sim 0.3 \mathrm{eV}$, much larger than thermal energies $(\sim 10 \mathrm{meV})$. States deeper into either the valence or conduction bands show progressively less thermal modulation because they are less localized (we have noted [15] a very strong correlation between the rms fluctuation in the energy eigenvalues due to thermal disorder and the inverse participation ratio, a simple measure of localization in the $\mathrm{T}=0$ model). The localization "amplifies" the electron-phonon coupling. Also, the conduction states fluctuate more than the valence states (suggesting that the conduction tails are more sensitive to thermal disorder than the valence tails which originate primarily from structural disorder), in pleasing agreement with total yield photoemission experiments [11] and earlier theory work [14].

Elsewhere [5], we have published color figures depicting the fluctuations in electron states near the gap for the system and dynamics described above. There are very substantial changes in the LUMO state in particular; There is a clear tendency for the LUMO state to alternately "accumulate" on a strained part of the network, sometimes becoming localized, but also occasionally developing a substantially more extended "stringlike" character. These are not the only two recognizable structures, but recur most frequently. The time between "characters" is not predictable, though it is of order tens to hundreds of fs. We have posted an animation of this state on the world wide web [16].

We have argued in the preceding section that structural disorder in a-Si gives rise to localized states with energies in the band tails. These system eigenstates can involve many atoms and can have a Byzantine [17] structure. The "simple physics" of this study is that the strong (compared to midband electrons) electron-phonon coupling for localized band tail states is sufficient to cause strongly time/temperature dependent quantum mechanical mixing of cluster states when the thermal disorder is "just right" to make their energies nearly degenerate provided that they have some overlap in real space. Strong mixing of course implies less localization and thus better prospects, at least while the more extended state survives, for conductivity and optical transitions. This work shows that transport and optical calculations based only on $\mathrm{T}=0$ results can be quite misleading. Mott [12] and others have made fundamental contributions to the theory of transport in a noncrystalline medium; for example,variable range hopping. In the kind of simulation we present here, I can estimate the conductivity, including its temperature and frequency dependence directly from the electronic states through an appropriate thermal average of the Kubo formula [18]. It is also a complement to the phenomenological models of transport $[13,19]$. In the latter work, transport is modeled as a hopping between localized tail states. My work can be viewed as an explanation of the precise nature of the states among 
which electrons are hopping (the very complicated states of Ref. 6). The waiting time between hops must be related to the time between eigenvalue "close encounters" near the Fermi level. It also points at an atomistic level to the dynamics of bandtail defects and their kinetics.

The consequences of this work can be stated another way. If $|i\rangle(|f\rangle)$ are initial (final) electronic states with energy $E_{i}\left(E_{f}\right)$, then for an electronic transition in a$\mathrm{Si}$, a Fermi golden rule argument leads quicky to the conclusion [12] that the transition rate is proportional to $|\langle i|\hat{T}| f\rangle|^{2} \delta\left(E_{f}-E_{i}-\hbar \omega\right)$, where $\hat{T}$ is a perturbation inducing the transition (to first approximation a momentum operator) and $\omega$ is the frequency of an external probe. Both the energies in the $\delta$ function and the transition matrix elements are affected by the instantaneous details of the structural disorder, and as such transition probabilities are also strongly dependent on the time and temperature. The consequences of this to transport are under investigation; the discussion here is based upon first-order time dependent perturbation theory, which for the very strong electron-phonon coupling we discuss, could be inadequate.

\section{Heuristics}

Earlier work has shown that it is useul to link the thermal fluctuation of the LDA energy eigenvalues near the band tails to the extent (localization) of the band tails in amorphous Si (as separately measured in total yield photoemission experiments). Here I give a simple model which makes this connection between fluctuating electron energies and lattice vibrations a bit more explicit.

Consider a particular electronic eigenvalue, $\lambda_{n}$, say in one of the band tails in a-Si. To estimate the sensitivity of $\lambda_{n}$ to a coordinate distortion (supposedly thermally induced), we can use the Hellmann-Feynman theorem [20], which gives $\partial \lambda_{n} / \partial \mathbf{R}_{\alpha}=\left\langle\psi_{n}\left|\partial \mathbf{H} / \partial \mathbf{R}_{\alpha}\right| \psi_{n}\right\rangle$ (for this to be valid, I must assume that the basis is fixed (not moving with the atoms) and that the $\left|\psi_{n}\right\rangle$ are exact eigenvectors of $\mathbf{H}$; see Ref. [8] for a more general case). Then clearly for small distortions $\mathbf{R}_{\alpha}$, we have $\delta \lambda_{n} \sim \sum_{\alpha}\left\langle\psi_{n}\left|\partial \mathbf{H} / \partial \mathbf{R}_{\alpha}\right| \psi_{n}\right\rangle \delta \mathbf{R}_{\alpha}$. Here, $\mathbf{R}$ is the $3 N$ vector of displacements for all of the atomic coordinates from equilibrium. If the displacements $\delta \mathbf{R}_{\alpha}(t)$ arise from classical vibrations, then one can also write $\delta \mathbf{R}_{\alpha}(t)=\sum_{\omega} A(T, \omega) \cos \left[\omega t+\phi_{\omega}\right] \chi_{\alpha}(\omega)$, where $\omega$ indexes the normal mode frequencies, $A(T, \omega)$ is the temperature dependent amplitude of the mode with frequency $\omega, \phi_{\omega}$ is an arbitrary phase, and $\chi_{\alpha}(\omega)$ is a normal mode with frequency $\omega$ and vibrational displacment index $\alpha$. Using a temperature dependent amplitude $A^{2}(T, \omega)=k_{B} T / 2 M \omega^{2}$, it is easy to see that the trajectory (long time) average of the expression for $\delta \lambda_{n}^{2}$ is: $\left\langle\delta \lambda_{n}^{2}\right\rangle \sim\left(k_{B} T / 4 M\right) \sum_{\omega}(\Xi(\omega) / \omega)^{2}$, where $\Xi(\omega)=\left[\sum_{\alpha}\left\langle\psi_{n}\left|\partial \mathbf{H} / \partial \mathbf{R}_{\alpha}\right| \psi_{n}\right\rangle \chi_{\alpha}(\omega)\right]$. It would be straightforward for a particular collection of vibrational states to include the correct Bose terms to obtain a result valid at low temperatures $T<\Theta_{D}$ (for $\Theta_{D}$ a salient De- bye temperature). These expressions give a transparent expression for the thermally induced electron modulation as driven by the lattice-electron coupling $\Xi(\omega)$. If we follow Ref. [14] and make the coarse approximation of equating $\left\langle\delta \lambda_{n}^{2}\right\rangle^{1 / 2}$ to the Urbach decay parameter [11], then this model would predict a square root dependence of the decay parameter with temperature, perhaps not easily distinguished from the linear dependence reported in Ref. [11].

It is easy to see why there is a correlation between an eigenstate's localization (as measured for example by inverse participation ratio) and its thermal rms fluctuation [15]. Note that $\Xi(\omega)$ will in general consist of a sum of many terms (for different $\alpha$ ); the individual terms have no preferred sign, so that adding a large number of terms of comparable magnitude will lead to cancellation and a small sum. On the other hand, if only a very small number of terms are nonzero (as for the case when the electron state $\left|\psi_{n}\right\rangle$ is well localized), then there will be less cancellation and a larger contribution to the sum (and therefore to the fluctuation of the eigenvalue). This model is limited in many ways (it is classical, as all the modes are equally populated from classical equipartition), it is obviously strictly harmonic and we are assuming no electronic level crossings or other departures from adiabatic (Born-Oppenheimer) dynamics [5]), but captures some of the right temperature dependent electronic effects observed.

The thermal effects on the electron states are more difficult to calculate with this approach, mostly because of problems with degeneracy if one tries to use perturbation theory. Still, the underlying "physics" is quite simple, just the effects of resonant mixing for close approach (in energy) of eigenvalues originating in cluster states with some overlap in space. In the language of the model Hamiltonian above, this means that there can be large mixing of cluster basis states when the energy denominator becomes small; here the thermal fluctations can induce small energy denominators (and therefore mixing). Of course a mixed state involves more cluster states than a pure cluster state, so that it is more spatially extended.

\section{Post adiabatic atomic dynamics}

The calculations and discussion of the preceding sections has been based on the adiabatic approximation (that the electrons are always in their instantaneous ground state determined by the external potential (eg the atomic nuclei), and that the forces "felt" by the nuclei arose from the instantaneous electronic structure of the system.

To see that this might be an imperfect assumption, consider a doped system with a small concentration of dopants for which the Fermi level is pushed into one one of the band tails. Then the thermal modulation of the atomic coordinates may produce level crossings or close approaches of the LDA energies. In the doped case, there would normally be (occupied) levels below 
the Fermi energy and (unoccupied) levels above. In the case of a level crossing at the Fermi level, the adiabatic approximation would immediately transfer charge from one state to another (even if spatially remote), which would induce changes in the dynamics (since the levels with changed occupation would typically be at least somewhat localized); and perhaps could lead to a structural change, even a long lived change if the new structure was "self-trapping". In practical calculations such charge transfer would not have be quite so large an affect, since one would usually smear the Fermi function slightly from the $T=0$ step form. The dynamics are then affected by such level crossings or close approaches even in the adiabatic approximation. A better theory [21] is due to Allen, in which interatomic forces are computed from mixed state wave functions obtained by a direct integration of the time-dependent Schrödinger equation (rather than the "pure" states computed anew at each new time step for the atomic dynamics in the adiabatic picture). This approach was formulated to model the dynamical response of a system to light (in which the simplest model of light-solid interactions would just involve promotions of electrons to low-lying conduction states), but the formalism is similar (and much simpler) for the case of purely phonon-induced level crossings as in our problem. It is possible in fact that the adiabatic approximation may often be satisfactory for this kind of problem, but the question has not yet been properly investigated because of difficulties with post-adiabatic calculations. One case for which nonadiabatic dynamics is essential is in the modeling of the thermalization of excited carriers to tail states. This process has never been realistically modeled despite its manifest importance to a host of problems in crystalline and non-crystalline semiconductor physics. More sophisticated methods for nonadiabatic dynamics are discussed in the quantum chemistry literature [22]; while fundamentally sound, these are currently too difficult to implement for the large model systems we must consider here.

\section{Conclusions}

We have used current computational techniques to demonstrate the nature of the localized to extended transition in amorphous $\mathrm{Si}$, and then took the additional step of adding thermal disorder and found that localized states could be time dependent. A simple analytic model was then presented, which explains some of the general temperature-dependent features observed in experiments. Finally we speculated about the importance of non-adiabatic dynamics to these calculations.

I note in closing that the effects reported here are presumably not peculiar to a-Si; they can be anticipated for any disordered insulator.

\section{Acknowledgements}

This work was supported in part by the National Science Foundation under grant DMR-96-18789. I thank my longtime collaborator Prof. Peter Fedders for many helpful discussions, and Dr. Jianjun Dong for his thesis work on the resonant cluster proliferation model.

\section{REFERENCES}

[1 ] See, for example, P. A. Fedders and D. A. Drabold, Phys. Rev. B 47 (1993) 13277, and references therein.

[2 ] P. A. Fedders, D. A. Drabold, P. Ordejón, G. Fabricius, D. Sanchez-Portal, E. Artacho and J. M. Soler (Phys. Rev. B in press, October 15, 1999)

[3 ] F. Wooten and D. Weaire, Solid State Physics, edited by H. Ehrenreich and D. Turnbull (Academic Press, New York, 1991), Vol. 40, p.2

[4 ] B. R. Djordjevic, M. F. Thorpe and F. Wooten, Phys. Rev. B 52 (1995) 5685.

[5 ] D. A. Drabold and P. A. Fedders, Phys. Rev. B 60 (1999) R721.

[6 ] Jianjun Dong and D. A. Drabold, Phys. Rev. Lett. 80 (1998) 1928.

[7 ] I. S. Duff, J. K. Reid, N. Munksgaard and H. B. Nielsen, J. Inst. Math. App. 23 (1979) 235; M. T. Jones and M. L. Patrick, SIAM J. Matrix Anal. App. 14 (1993) 553.

[8 ] O. F. Sankey and D. J. Niklewski, Phys. Rev. B 40 (1989) 3979; A. A. Demkov, J. Ortega, O. F. Sankey and M. Grumbach, Phys. Rev. B 52 (1995) 1618.

[9 ] C. Herring, Phys. Rev. 57 (1940) 1169.

[10 ] U. Stephan and D. A. Drabold, Phys. Rev. B 57 (1998) 6391; U. Stephan, R. M. Martin and D. A. Drabold, Extended range computation of Wannier functions in amorphous semiconductors, submitted to Nature.

[11 ] S. Aljishi, J. D. Cohen, and L. Ley, Phys. Rev. Lett. 64 (1990) 2811.

[12 ] N. F. Mott and E. A. Davis, Electronic processes in non-crystalline materials, 2nd ed. (Clarendon, Oxford, 1979).

[13 ] V. I. Arkhipov and G. J. Adriaenssens, J. NonCryst. Sol. 227 (1998) 166; Phys. Rev. B 54 (1996) 16696.

[14 ] D. A. Drabold, P. A. Fedders, S. Klemm and O. F. Sankey, Phys. Rev. Lett. 67 (1991) 2179.

[15 ] M. Cobb and D. A. Drabold, Phys. Rev. B 56 (1997) 3054. 
[16 ] For an mpeg format animation, see: http://www.phy.ohiou.edu/ drabold/fluctuate.html

[17 ] E. Gibbon, The Decline and Fall of the Roman Empire, abridged edition by D. M. Low, (Harcourt Brace, New York, 1960).

[18 ] D. A. Drabold in Insulating and semiconducting glasses edited by P. Boolchand (World Scientific, Singapore, 1999).

[19 ] See, for example P. Thomas and S. D. Baranovskii, J. Non-Cryst. Sol. 164, (1996) 431 and references therein.

[20 ] R. P. Feynman, Phys. Rev. 56(1939) 340; H. Hellmann, Einfuhrung in die Quantumchemie (Franz Deutsche, Leipzig, 1937)

[21 ] R. E. Allen, Phys. Rev. B 50 (1994) 18629.

[22 ] See for example, K. Thompson and T. J. Martinez, J. Chem. Phys. 110 (1999) 1376 and references therein. 